\title{
Nano-scale Crystallographic Aspects of Discontinuous Precipitation and Coarsening Reactions in Cu-10\% Co Alloy
}

\author{
N. M. Suguihiro ${ }^{1,2 *}$ I. G. Solórzano ${ }^{2,3}$ \\ 1. Campus Duque de Caxias, Universidade Federal do Rio de Janeiro, Rio de Janeiro, Brazil \\ 2. DEQM, Pontifícia Universidade Católica do Rio de Janeiro, Rio de Janeiro, Brazil \\ 3. SEAS, Harvard University, Cambridge, USA \\ *Corresponding author: natasha@caxias.ufrj.br
}

Discontinuous precipitation (DP) and discontinuous coarsening (DC) are phase transformations in which the formation of a new phase occurs simultaneously with GB migration. DP Take place in hundreds of metallic systems, being responsible for drastic changes in materials properties. This phenomenon occurs in supersaturated alloys in conditions in which GB migrate dragging solute, and leaving behind aggregates of lamellar or rod-like precipitates, depending on crystallographic matrix/precipitate relationships. After the end of DP, if $\alpha / \beta$ interface energy is very high and/or there still a remaining supersaturation in between discontinuous precipitates, GB reaction front moves toward discontinuous precipitates, dissolving them and leaving behind coarser but still lamellar or rod-like precipitates, in a process known as discontinuous coarsening (DC). One of the factors with great impact on the development of DP and DC is GB structural properties such as structural inclination/misorientation, and orientation relationships (OR) between GB and precipitated phases. Such differences have direct influence in the incubation time required for DP initiation, and could even suppress DP and DC development [1].

In this research we studied crystallographic aspects of DP and DC in $\mathrm{Cu}-10 \%$ Co alloy by means of automated crystalline orientation and phase maps, allowed by nano beam electron diffraction in the scanning transmission electron microscope. The instrument employed was a TEM/STEM JEOL JEM 2100F of LabNano/CBPF, operating at 200kV, equipped with automated precession electron diffraction NanoMEGAS ASTAR/DigiSTAR system for acquisition of nano beam diffraction (NBD) patterns, with zero degrees of precession and electron probe of $2 \mathrm{~nm}$. Results suggest that GB mobility of as solidified alloy is poorly affected by orientation of adjacent GB. Figure 1a is a virtual bright field image of the analyzed area, which comprises several sub-micron grains that developed DP and DC upon isothermal aging treatment. The bulging of GB by DC rod-like Co precipitates is clear NBD pattern of the point marked with a red cross in Figla is shown in a detail of Fig. 1b, with a virtual dark field image constructed by the selected spot marked on DP. Notice the bright spots from homogeneous precipitates. Fig. 1c is the generated orientation map. Notice also the no-preferential direction for GB migration. The GB marked by a square seems to have no mobility, but instead rod-like precipitates pushes it in the direction of the adjacent grain. This effect is associated to chemical forces due to differences in chemical potential across precipitate/matrix interfaces which will later result in the migration of GB since it generates capillary forces between the precipitates.

In a later stage of DC, after longer annealing times, it was found that rod-like precipitates from discontinuous coarsening generates incoherent interfaces. It is reported that original DP precipitates in this alloy system nucleates and grow with coherent interfaces, losing coherence during continuous coarsening [2]. Fig. 2a shows a DC colony with incoherent DC precipitate, clearly identified by Co EDS elemental mapping of a rod-like precipitate shown in Figs.2b and c. Fig $2 d$ is a virtual bright field image of NBD scanned area, and Fig2e is the orientation map of the same region. EDS mapping shown in Fig. 2c, corroborated by phase map of Fig.2e, show that this particular rod-like precipitate is losing its elongated morphology due to a continuous coarsening due to the remaining supersaturation in the matrix. By orientation map one could identify a rotation misalignment, with $001 / / 101$ matrix/precipitate orientation. This is unexpected fact since both phases 
have $f c c$ structure. There are reports on literature suggesting a twin-like type of OR instead of cube-cube relationship of precipitate/matrix in annealed melt-spun $\mathrm{Cu}-70 \% \mathrm{Co}$ alloy [3], which lead us to believe that this is the case for DC precipitates. Other authors [4] have reported that Co coherent precipitates tends to develop octahedron-like morphology with $\{110\}$ facets while after coherency loss have $\{111\}$ facets, justifying these changes by means of magnetic interactions.

\section{References:}

[1] I Manna, SK Pabi, W Gust, Int Mater Rev 46 (2001), p. 53.

[2] NM Suguihiro, IG Solórzano, J Mat Sci 50 (2016) p. 71.

[3] X Song, SW Mahon, RF Cochrane, Mat Res Bull 32 (1997), p. 485.

[4] M Takeda et al., Phys Stat Sol 168 (1998), p. 27.
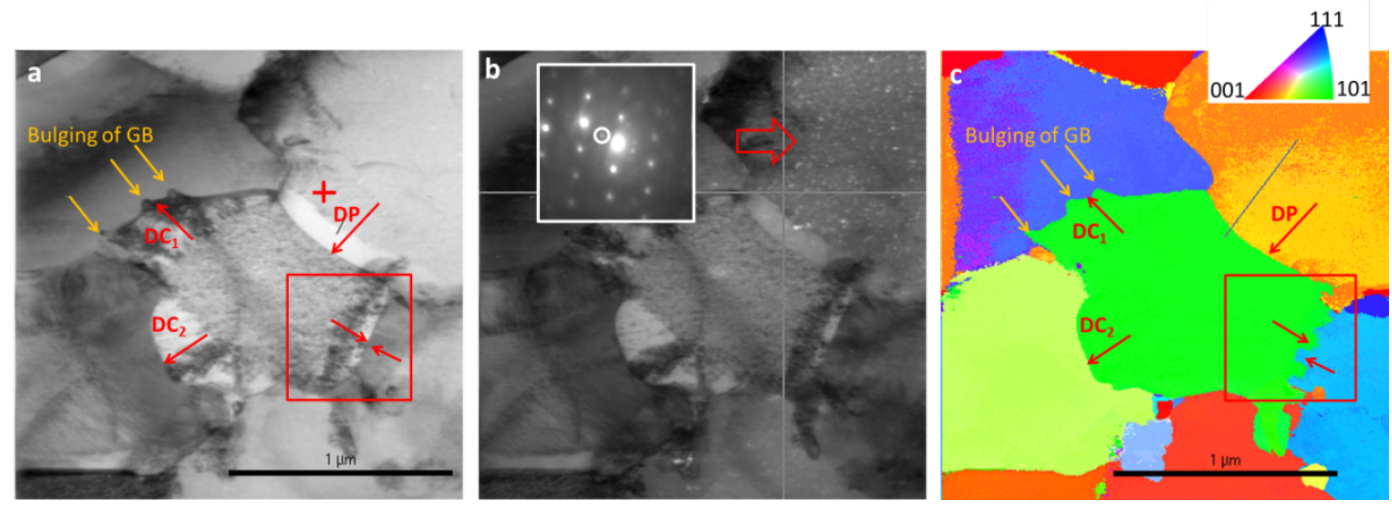

Figure 1. Nano-beam diffraction analysis of $\mathrm{Cu}-10 \% \mathrm{Co}$ annealed at $550^{\circ} \mathrm{C}$ for $30 \mathrm{~min}$. (a) Bright field (BF) virtual image (b) Dark field virtual image from the spot marked in the inserted DP. This DP is referent to the point marked with the red cross. (c) Orientation map in which are marked 3 different DC and a DP colony.

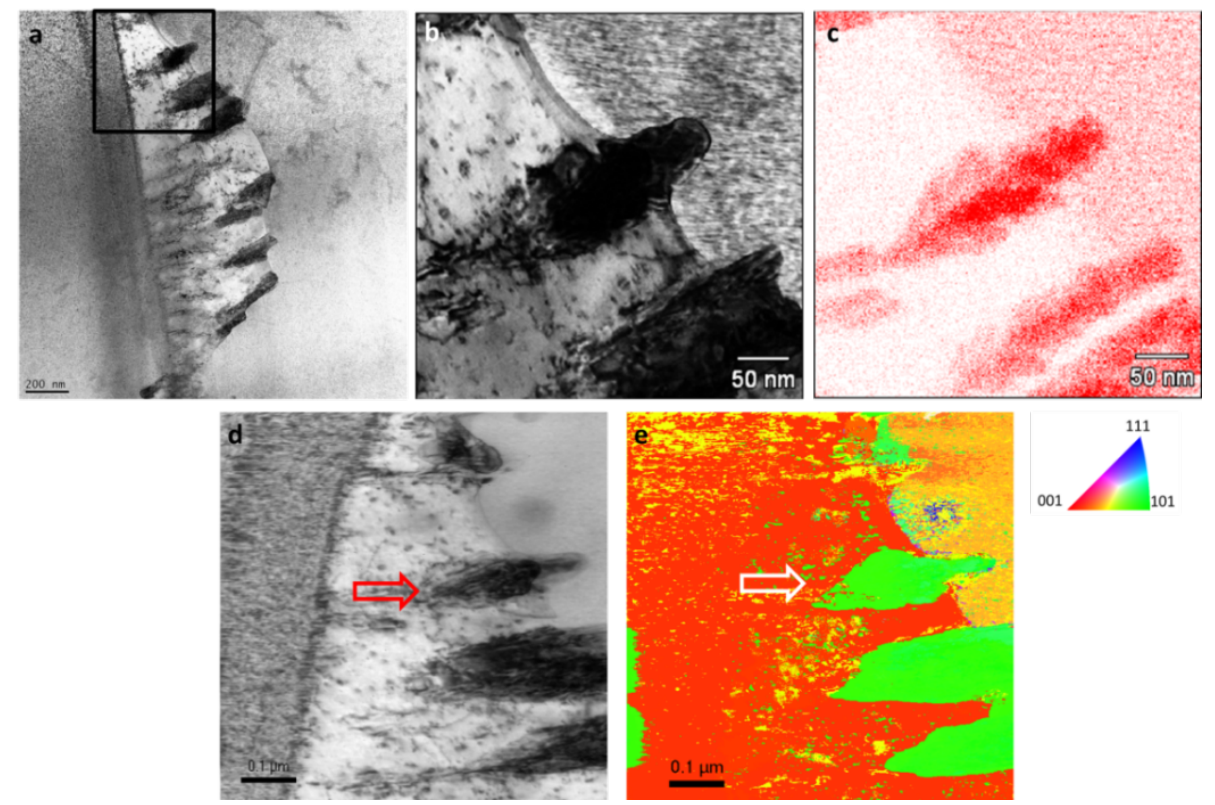

Figure 2. (a) BF STEM of a well-developed DC colony after annealing at $650^{\circ} \mathrm{C}$ for $60 \mathrm{~min}$. (b) BF STEM of precipitate marked in Fig.2a, and (c) Co EDS mapping of incoherent rod-like precipitates. (d) BF virtual image of nano-beam scanning diffraction of the same DC colony and (e) orientation map. Notice the precipitate/matrix orientation on 101//001 direction. 